\title{
Apoptosis and Cell Cycle Arrest in Two Human Breast Cancer Cell Lines by Dieckol Isolated from Ecklonia cava
}

\author{
Sun Hyong You, Jeong-Soo Kim, Yong-Seok Kim \\ Department of Surgery, College of Medicine, The Catholic University of Korea, Seoul, Korea
}

Purpose: Dieckol, a phlorotannin compound isolated from Ecklonia cava, has been reported to have antioxidant, antiviral, anti-inflammatory, and anticancer properties. The purpose of this study was to investigate its anticancer effects on human breast cancer cell lines. Methods: In this study, the viability of two human breast cancer cell lines SK-BR-3 and MCF-7 was investigated after dieckol treatment using a WST-1 assay. Apoptosis and cell cycle distribution were assayed via Annexin V-fluorescein isothiocyanate and propidium iodide staining followed by flow cytometric analysis. Immunoblotting analysis was also performed using Bax/Bcl-2 to determine whether the dieckol-induced apoptosis was mediated by the intrinsic apoptotic pathway. Results: In a dose dependent manner, dieckol reduced the number of viable cells and increased the number of apoptotic cells. The effect of dieckol on the cell cycle distribution was analyzed using flow cytometry. Dieckol treatment significantly increased the percentage of MCF-7 and SK-BR-3 in the G2/M phase. Immunoblot analysis revealed that 24 hours of dieckol exposure increased the Bax/Bcl-2 ratio. Conclusion: Dieckol induced cytotoxicity in MCF-7 and SK-BR-3 human breast cancer cells inducing apoptosis and cell cycle arrest. Therefore, it is suggested that dieckol may be a potential therapeutic agent for breast cancer.

Key Words: Apoptosis, Breast neoplasms, Cell cycle checkpoints, Dieckol

\section{INTRODUCTION}

With advances in treatment, earlier detection, and the increased awareness of health care, the mortality rate due to breast cancer has been decreasing over the last few decades [1]. However, breast cancer is still a major cause of morbidity and mortality among women worldwide. Moreover, it is the second most common cancer in Korean women [2]. Generally, the treatment of breast cancer consists of surgery, chemotherapy, radiation therapy, antihormonal therapy, and/or targeted therapy (trastuzumab). These treatment strategies vary for each patient, depending on the breast cancer subtype. Since breast cancer is a disease characterized by multiple subtypes, there are differences in disease prognosis and the response to diverse therapeutic options [3-5]. The recurrence of breast cancer can occur at any time, from months to years after the initial treatment. Late recurrence is not common in breast cancer but may arise at any stage [6]. Prognosis is poor in patients with recurrent or metastatic breast cancer, despite adequate treatments such as chemotherapy or radiation therapy. Additionally,

\section{Correspondence: Yong-Seok Kim}

Department of Surgery, Uijeongbu St. Mary's Hospital, College of Medicine, The Catholic University of Korea, 271 Cheonbo-ro, Uijeongbu 11765, Korea Tel: +82-31-820-3817, Fax: +82-31-847-2717, E-mail: dydtjr97@catholic.ac.kr Received: May 16, 2018 Revised: Jul 16, 2018 Accepted: Aug 28, 2018 cancer treatment is known to cause adverse effects including febrile neutropenia, hair loss, peripheral neuropathy, ototoxicity, and nephrotoxicity [7-9]. These events decrease the patient's compliance to treatment. Therefore, new therapeutic options are needed with consideration of the patient's compliance and quality of life.

Extensive research has revealed that numerous natural products, especially those obtained from aquatic plants, have potential chemopreventive and antineoplastic effects on breast cancer [10,11]. Among these, brown algae, i.e., Ecklonia cava, are used as health foods in East Asia. In addition, they are used in the production of many food products $[12,13]$. E. cava contains various natural components, such as carotenoids, fucoidans, and phlorotannins. Dieckol, a phlorotannin from E. cava has antioxidant, antithrombotic, profibrinolytic properties $[14,15]$. Recent studies have reported that this phlorotannin has an inhibitory effect on cancer metabolism [16,17]. However, the effect of dieckol on cancer cell growth has not been extensively studied in breast cancer.

Therefore, in this study, the effect of dieckol isolated from E. cava was evaluated on human breast cancer cell growth. Further, its mechanism of action on the cell lines, i.e., induction of apoptosis and/or cell cycle arrest was investigated. 


\section{METHODS}

\section{Cell lines and culture}

Human breast adenocarcinoma (MCF-7 and SK-BR-3) cells were purchased from the American Tissue Culture Collection (ATCC, Manassas, USA). These cells were cultured in Dulbecco's modified Eagle medium: Nutrient Mixture F-12 (DMEM/F-12) supplemented with $10 \%$ fetal bovine serum and $1 \%$ Penicillin-Streptomycin. The cells were maintained at $37^{\circ} \mathrm{C}$ in a $5 \% \mathrm{CO}_{2}$ incubator. Dieckol was solubilized in dimethyl sulfoxide and then diluted in DMEM/F-12 medium for cell treatment.

\section{Chemical treatment and cell viability assay}

MCF-7 and SK-BR-3 cells were seeded in 96-well plates at a density of 1,000 cells/well in $100 \mu \mathrm{L}$ of medium and permitted to adhere for 24 hours at $37^{\circ} \mathrm{C}$. The cells were treated with various concentrations of the compound $(25,50,100,200$, and $400 \mu \mathrm{g} / \mathrm{mL})$ and incubated for 24 hours at $37^{\circ} \mathrm{C}$. The cell viability was evaluated using a WST-1 kit (Roche Diagnostics GmbH, Mannheim, Germany) according to the manufacturer's protocol. In brief, $10 \mu \mathrm{L}$ of the WST-1 reagent was added to the wells, and the cells were incubated at $37^{\circ} \mathrm{C}$ for 3 hours. The cells were analyzed using a plate reader at a wavelength of 440 $\mathrm{nm}$. The results are from experiments repeated at least in triplicate.

\section{Sodium dodecyl sulfate-polyacrylamide gel} electrophoresis and immunoblot assay

The protein samples were balanced, and approximately $20 \mu \mathrm{g}$ of protein was separated on $10 \%$ to $18 \%$ acrylamide gels and then transferred to polyvinylidene difluoride membranes (GE Healthcare, Mississauga, Canada). The membranes were blocked with 5\% nonfat dried milk for 1 hour and incubated overnight at $4^{\circ} \mathrm{C}$ with the appropriate primary antibodies (Sigma Aldrich, Cell Signaling, Darmstadt, Germany). The secondary antibodies used were either goat anti-rabbit or anti-mouse IgG (Bio-Rad, Seoul, Korea) conjugated to horseradish peroxidase. A chemiluminescent substrate (GE Healthcare) was used to visualize the immunoreactive proteins.

\section{Annexin $\mathrm{V}$ and propidium iodide double staining for the analysis of apoptosis}

For the analysis of cell apoptosis, cells were treated with $100 \mu \mathrm{g} / \mathrm{mL}$ of dieckol for 24 hours at $37^{\circ} \mathrm{C}$. The cells were collected and stained using the fluorescein isothiocyanate (FITC) Annexin V Apoptosis Detection Kit I (BD Biosciences, Seoul, Korea) according to the manufacturer's protocol. In brief, the cells were seeded in 6-well plates at a density of $3 \times 10^{5}$ cells/well in $2 \mathrm{~mL}$ of medium and were permitted to adhere for 24 hours at $37^{\circ} \mathrm{C}$. The cells were treated with dieckol and incubated for 24 hours at $37^{\circ} \mathrm{C}$. The cells were harvested in a $5-\mathrm{mL}$ flow cytometry (FACS) tube and washed with cold phosphate-buffered saline (PBS). FITC-Annexin V and propidium iodide (PI) were both added, and the solution incubated for 15 minutes at room temperature $\left(25^{\circ} \mathrm{C}\right)$ in the dark. Samples were analyzed by FACS (BD Biosciences)

\section{Assays of cell cycle}

For the analysis of the cell cycle, the dieckol-treated cells were collected and fixed with $70 \%$ ice-cold ethanol for 1 hour at $4^{\circ} \mathrm{C}$. The cells were subsequently washed once with PBS. They were then incubated in PBS with RNase $(0.5 \mathrm{mg} / \mathrm{mL})$ for 1 hour at $37^{\circ} \mathrm{C}$. Further, the cells were then incubated with PI for 30 minutes at room temperature $\left(25^{\circ} \mathrm{C}\right)$ in the dark. The samples were analyzed by FACS (BD Biosciences) at $488 \mathrm{~nm}$.

\section{RESULTS}

\section{Dieckol suppresses breast cancer cell growth}

The inhibitory effect of dieckol on MCF-7 and SK-BR-3 cell growth was investigated. These cells were exposed to various concentrations of dieckol for 24 hours, and the cell viability was assessed using a WST-1 assay. The results showed a dose-dependent effect on cell viability. Cell death reached $57.55 \%$ with $400 \mu \mathrm{g} / \mathrm{mL}$ of dieckol and $15.40 \%$ with $200 \mu \mathrm{g} / \mathrm{mL}$ of dieckol after 24 hours in MCF-7 cells. Similar results were observed in the SK-BR-3 cells. Cell death reached $63.29 \%$ with $400 \mu \mathrm{g} / \mathrm{mL}$ of dieckol and $40.89 \%$ with $200 \mu \mathrm{g} / \mathrm{mL}$ of dieckol in comparison with the control (Figure 1).

\section{Apoptosis induced by dieckol}

To investigate the mechanism of the cell death induced by this compound, apoptosis was analyzed. The results are presented in Figure 2. These cells were treated with a fixed concentration of dieckol $(100 \mu \mathrm{g} / \mathrm{mL})$. Flow cytometric assay showed different results between 

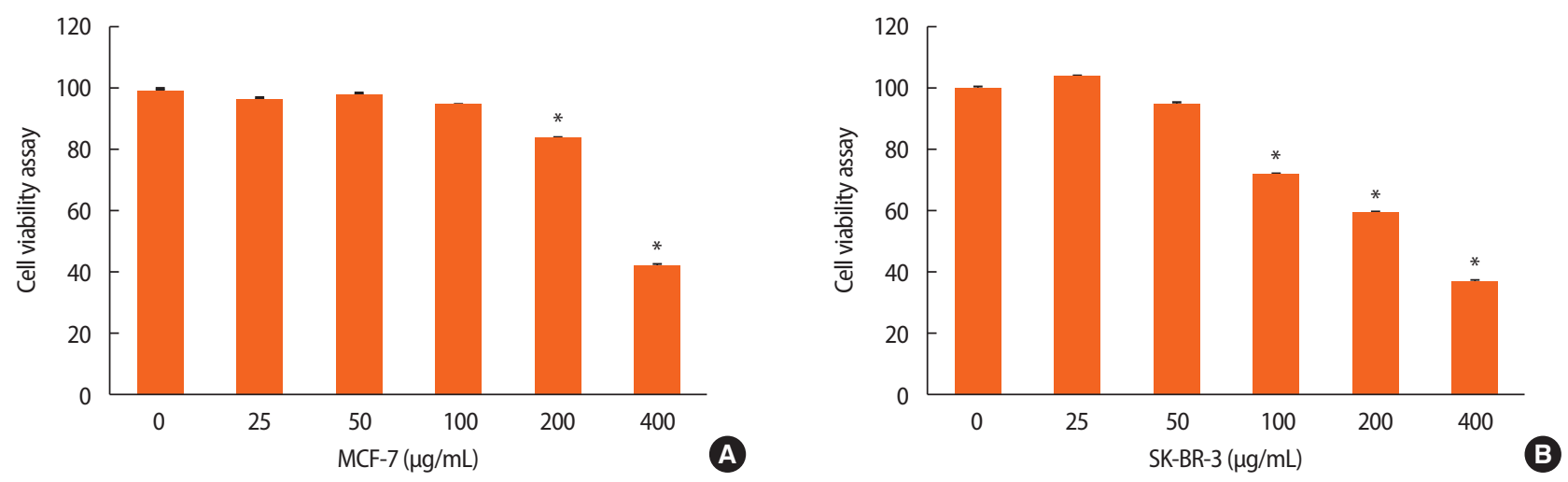

Figure 1. Effect of dieckol on the proliferation of MCF-7 (A) and SK-BR-3 (B) cells by WST-1 assay. *Significant difference from control $p<0.001$.
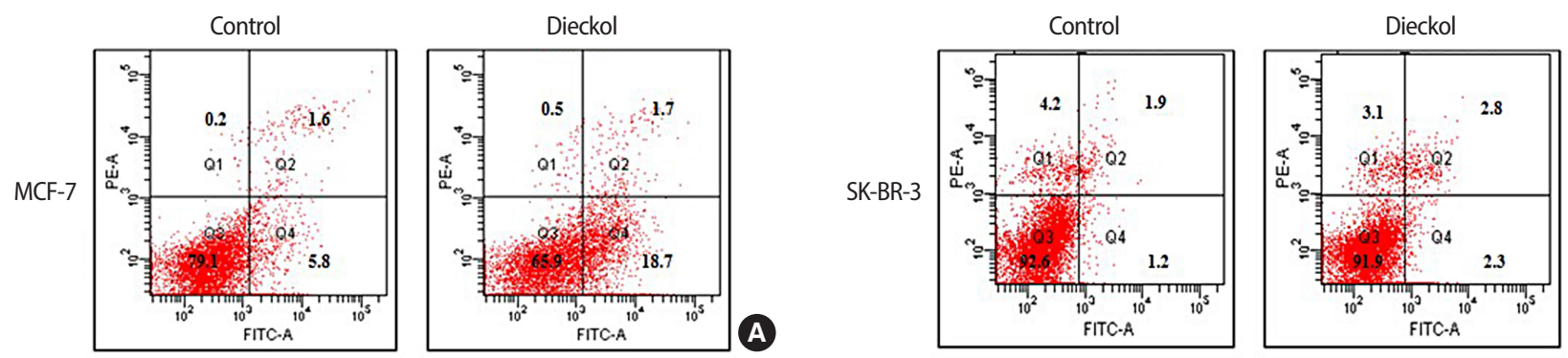

Figure 2. Flow cytometric analysis of the effect of dieckol on apoptosis of MCF-7 (A) and SK-BR-3 (B) cells. Both cells were treated with $100 \mu \mathrm{g} / \mathrm{mL}$ of dieckol for 24 hours.

$\mathrm{PE}-\mathrm{A}=$ phycoerythrin; FITC=fluorescein isothiocyanate.
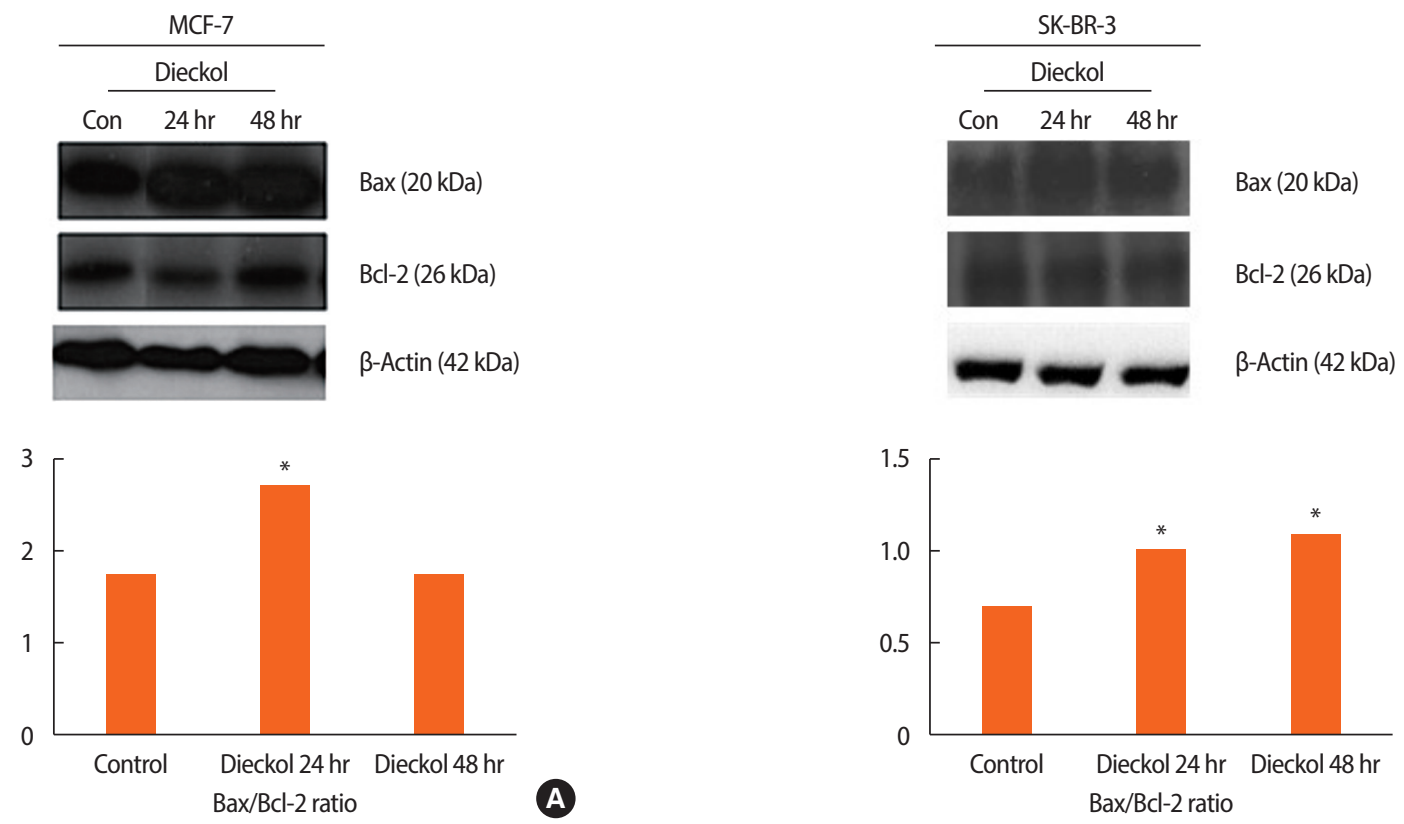

Figure 3. Bax/Bcl-2 ratio measured by western blot analysis. Three groups are shown: control (con); $100 \mu \mathrm{g} / \mathrm{mL}$ dieckol for 24 hours; $100 \mu \mathrm{g} / \mathrm{mL}$ dieckol for 48 hours. (A) In MCF-7 cell, representative gel pictures showing level of expression Bax, BCl-2, and $\beta$-actin densitometric analysis showing three groups. (B) In SK$\mathrm{BR}-3$ cell, representative gel pictures showing level of expression of $\mathrm{Bax}, \mathrm{BCl}-2$, and $\beta$-actin densitometric analysis showing three groups.

*Significant difference from control $p<0.001$. 
the cell lines. Dieckol-induced apoptosis was significantly higher in MCF-7 cells than in SK-BR-3 cells. The percentage of apoptotic cells increased from $5.8 \%$ to $18.7 \%$ in the MCF-7 cell line and from $1.2 \%$ to $2.3 \%$ in the SK-BR-3 cell line.

\section{$\mathrm{Bax} / \mathrm{Bcl}-2$ ratio in the breast cancer cell lines treated} with dieckol

To determine the amount of dieckol-induced apoptosis through the modulation of the $\mathrm{Bcl}-2$ proteins, the $\mathrm{Bax} / \mathrm{Bcl}-2$ ratios were measured at the protein level. These ratios in the MCF-7 and SK-BR-3 cells were determined at 24 and 48 hours after treatment with dieckol (100 $\mu \mathrm{g} / \mathrm{mL}$ ). The expression of $\beta$-actin $(42 \mathrm{kDa})$ was used as a loading control. The $\mathrm{Bax} / \mathrm{Bcl}-2$ ratio was higher in the dieckol-treated group than in the control at 24 hours $(p<0.001)$ (Figure 3A). However, it was observed that in the MCF-7 cell line, this ratio was not significantly different between the control and the dieckol-treated group at 48 hours.

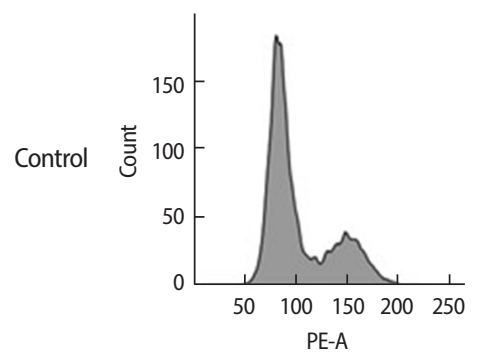

SubG1 $\quad 0.077$

G0/G1 68.9

$\mathrm{S} \quad 6.05$

G2/M 24.8

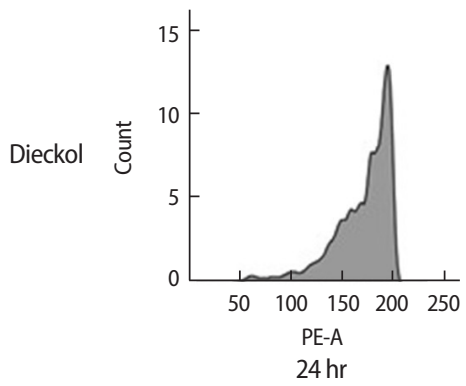

SubG1 $\quad 0.21$

G0/G1 5.41

$\mathrm{S} \quad 3.95$

G2/M $\quad 90.2$

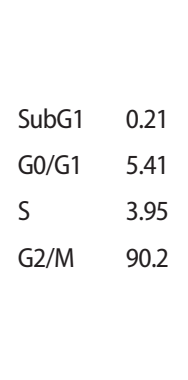

MCF-7

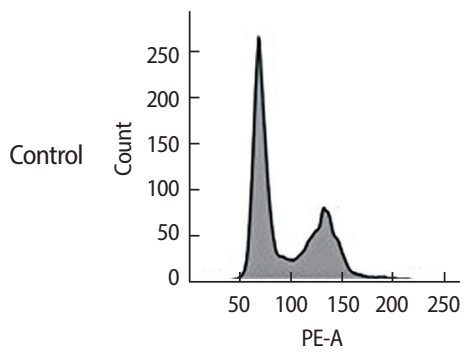

SubG1 $\quad 0.49$

G0/G1 55.1

S $\quad 6.07$

G2/M $\quad 36.7$

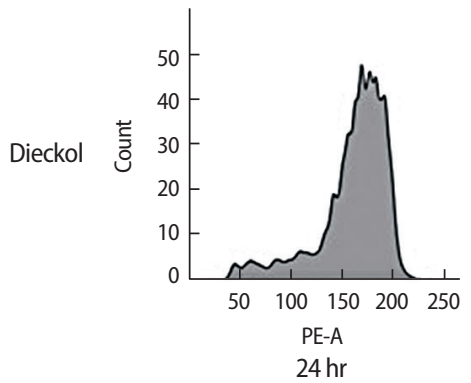

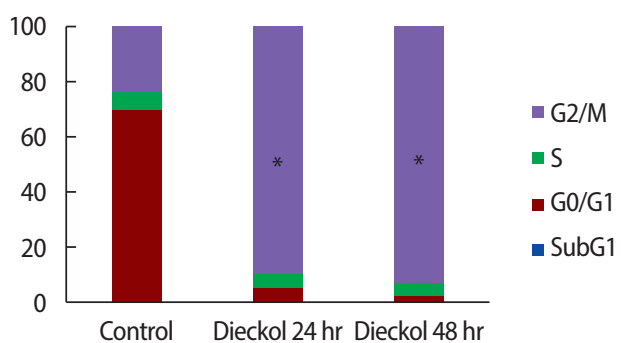

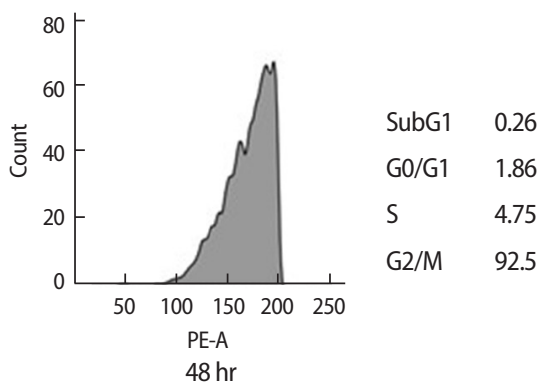

A

SK-BR-3
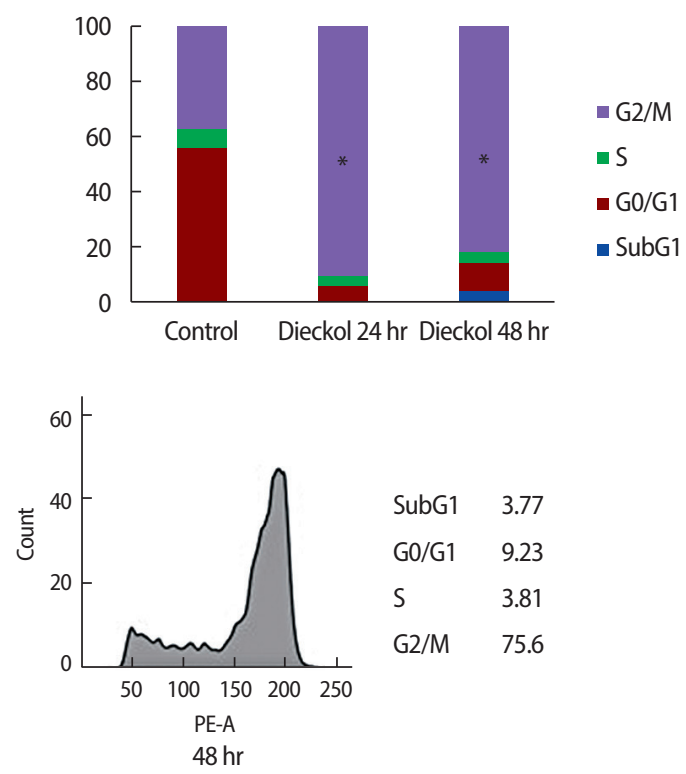

Figure 4. Dieckol induces cell cycle arrest at the G2/M phase in MCF-7 (A) and SK-BR-3 (B) cells. The cell cycle was measured by flow cytometry. $\mathrm{PE}-\mathrm{A}=$ phycoerythrin. ${ }^{*}$ Significant difference from control $p<0.001$. 
The ratios measured in SK-BR-3 cells are displayed in Figure 3B. There was a significant difference $(p<0.001)$ in the $\mathrm{Bax} / \mathrm{Bcl}-2$ ratio between the cells treated with dieckol for 24 and 48 hours.

\section{Cell cycle arrested induced by dieckol}

The effect of dieckol on the cell cycle distribution was analyzed using flow cytometry. As shown in Figure 4A, in the dieckol-treated groups, the percentage of MCF-7 cells in the S phase decreased. On the other hand, there was an increase in the percentage of cells in the G2/M phase. These results showed that dieckol induced cell cycle arrest at the G2/M phase in MCF-7 cells.

Figure $4 \mathrm{~B}$ shows the results of the cell cycle analysis of the SK-BR-3 cells with and without dieckol treatment. Compared to the control group, the dieckol significantly increased the percentage of SK-BR-3 cells in the $\mathrm{G} 2 / \mathrm{M}$ phase.

\section{DISCUSSION}

Marine brown algae, E. cava, are abundant on the Jeju island of Korea and Japan. This species has been found to contain various phlorotannins, such as fucodiphlorethol G, 7-phloro eckol, 6,6'-bieckol, eck$\mathrm{ol}$, dieckol, and phlorofucofuroeckol A [18-20]. These components are known to have strong antioxidant, antibacterial, anti-inflammatory, antidiabetic, and anticancer activities [16,21-23]. Most studies related to the anticancer effect of dieckol have been performed by Korean researchers. This phenomenon may occur because it dieckol is an affordable ingredient in Korea. However, studies regarding its anticancer effects have not been reported. To the best of our knowledge, this is the first study that has investigated the anticancer effects of dieckol, such as apoptosis and cell cycle arrest, using two breast cancer cell lines (MCF-7 and SK-BR-3). These cells exhibit different phenotypes of breast cancer. MCF-7 is hormone-receptor positive and human epidermal growth factor receptor 2 (HER2) negative, whereas SK-BR-3 is hormone-receptor positive and overexpresses HER2. Although MCF7 and SK-BR-3 are different, the anticancer effect of dieckol did not vary between the two cell lines.

In this study, since the exact dieckol concentration required to cause cancer cell death was unknown, various concentrations were applied. It was observed that dieckol was highly effective at killing the MCF-7 and SK-BR-3 breast cancer cell lines in a dose-dependent manner. There was a significant decrease in the proliferation of both the cancer cell lines treated with $200 \mu \mathrm{g} / \mathrm{mL}$ of dieckol for 24 hours compared to the control. Therefore, dieckol could cause cytotoxicity in breast cancer cells.

Apoptosis consists of two major pathways, the extrinsic and intrinsic pathways. The intrinsic pathway, i.e., the mitochondrial-mediated pathway, is activated by various stimuli, such as reactive oxygen species, DNA damage, calcium, and Bcl-2 antiapoptotic proteins. Moreover, the mitochondrial-mediated pathway is regulated by the antiapoptotic Bcl-2 family and the proapoptotic Bax protein. Bcl-2 inhibits apoptosis by interacting with and forming inactivating heterodimers with Bax/Bak $[24,25]$. To investigate whether apoptosis was induced by the mitochondrial-mediated pathway, the $\mathrm{Bax} / \mathrm{Bcl}-2$ ratio in the breast cancer cell lines treated with dieckol was measured. It was revealed that the $\mathrm{Bax} / \mathrm{Bcl}-2$ ratio increased in both cell lines treated with dieckol for 24 hours. These results indicate that the dieckol-induced apoptosis may be initiated by the mitochondrial pathway. Interestingly. there was no increase in the $\mathrm{Bax} / \mathrm{Bcl}-2$ ratio in the MCF-7 cells treated with dieckol for 48 hours, which is in contrast with the result of SK-BR-3 cells treated for the same amount of time. The reason for this discrepancy in the $\mathrm{Bax} / \mathrm{Bcl}-2$ ratio in both cell lines treated with dieckol for 48 hours could be because they are different cell types. However, this finding warrants further investigation.

The cell cycle is a set of events responsible for cell replication [26]. In normal cells, the cell cycle is controlled by various signaling pathways that detect cellular events, such as cellular stress or DNA damage. These pathways are generally referred to as cell cycle checkpoints. These checkpoints play a role in correcting and repairing cellular damage, cell cycle arrest, and apoptosis [27,28]. In tumor metabolism, uncontrolled cell proliferation is observed because of malfunctions in the regulatory processes. In addition, evading cell cycle arrest is the most frequently observed phenomenon. Checkpoints at the G1/S and G2/M transitions are crucial to cell cycle progression without accumulation of genetic abnormalities [29,30]. In this study, the MCF-7 and SK-BR-3 cells treated with a concentration of $100 \mu \mathrm{g} / \mathrm{mL}$ of dieckol for 48 hours were arrested at the G2/M phase. These findings indicate that the dieckol-induced cell cycle arrest occurred regardless of the cell line. However, the precise mechanisms of the cell cycle arrest in the MCF-7 and SK-BR-3 cells are unknown. Therefore, further investigation is needed. 
In conclusion, this study indicated that dieckol suppresses cell proliferation, activates the intrinsic apoptotic pathway, and induces cell cycle arrest at the G2/M phase. Although further investigations are required to be conducted in vivo, these findings can support the development of dieckol as a potential agent for breast cancer treatment.

\section{CONFLICT OF INTEREST}

The authors declare that they have no competing interests.

\section{ACKNOWLEDGMENTS}

Dieckol was supported by Botamedi, Inc., Seoul, Korea

\section{REFERENCES}

1. DeSantis C, Ma J, Bryan L, Jemal A. Breast cancer statistics, 2013. CA Cancer J Clin 2014;64:52-62.

2. Lee JH, Shim JW, Choi YJ, Heo K, Yang K. The combination of sorafenib and radiation preferentially inhibits breast cancer stem cells by suppressing HIF-1 a expression. Oncol Rep 2013;29:917-24.

3. Perou CM, Sørlie T, Eisen MB, van de Rijn M, Jeffrey SS, Rees CA, et al. Molecular portraits of human breast tumours. Nature 2000; 406:747-52.

4. Sørlie T, Perou CM, Tibshirani R, Aas T, Geisler S, Johnsen H, et al. Gene expression patterns of breast carcinomas distinguish tumor subclasses with clinical implications. Proc Natl Acad Sci U S A 2001;98:10869-74.

5. Sotiriou C, Neo SY, McShane LM, Korn EL, Long PM, Jazaeri A, et al. Breast cancer classification and prognosis based on gene expression profiles from a population-based study. Proc Natl Acad Sci U S A 2003;100:10393-8.

6. Omidvari S, Hamedi SH, Mohammadianpanah M, Nasrolahi H, Mosalaei A, Talei A, et al. Very late relapse in breast cancer survivors: a report of 6 cases. Iran J Cancer Prev 2013;6:113-7.

7. Tew WP, Muss HB, Kimmick GG, Von Gruenigen VE, Lichtman SM. Breast and ovarian cancer in the older woman. J Clin Oncol 2014;32:2553-61.

8. Lemieux J, Provencher L, Laflamme C. Survey about the use of scalp cooling to prevent alopecia during breast cancer chemothera- py treatment in Canada. Can Oncol Nurs J 2014;24:102-8.

9. Miller RP, Tadagavadi RK, Ramesh G, Reeves WB. Mechanisms of Cisplatin nephrotoxicity. Toxins (Basel) 2010;2:2490-518

10. Bonofiglio D, Giordano C, De Amicis F, Lanzino M, Andò S. Natural products as promising antitumoral agents in breast cancer: mechanisms of action and molecular targets. Mini Rev Med Chem 2016;16:596-604.

11. Vadodkar AS, Suman S, Lakshmanaswamy R, Damodaran C. Chemoprevention of breast cancer by dietary compounds. Anticancer Agents Med Chem 2012;12:1185-202.

12. Wijesinghe WA, Jeon YJ. Exploiting biological activities of brown seaweed Ecklonia cava for potential industrial applications: a review. Int J Food Sci Nutr 2012;63:225-35

13. Kim SK, Lee DY, Jung WK, Kim JH, Choi I, Park SG, et al. Effects of Ecklonia cava ethanolic extracts on airway hyperresponsiveness and inflammation in a murine asthma model: role of suppressor of cytokine signaling. Biomed Pharmacother 2008;62:289-96.

14. Kang HS, Chung HY, Kim JY, Son BW, Jung HA, Choi JS. Inhibitory phlorotannins from the edible brown alga Ecklonia stolonifera on total reactive oxygen species (ROS) generation. Arch Pharm Res 2004;27:194-8

15. Kim TH, Ku SK, Bae JS. Antithrombotic and profibrinolytic activities of eckol and dieckol. J Cell Biochem 2012;113:2877-83.

16. Ahn JH, Yang YI, Lee KT, Choi JH. Dieckol, isolated from the edible brown algae Ecklonia cava, induces apoptosis of ovarian cancer cells and inhibits tumor xenograft growth. J Cancer Res Clin Oncol 2015;141:255-68.

17. Kim EK, Tang Y, Kim YS, Hwang JW, Choi EJ, Lee JH, et al. First evidence that Ecklonia cava-derived dieckol attenuates MCF-7 human breast carcinoma cell migration. Mar Drugs 2015;13:1785-97.

18. Lee SM, Bae SK, Kim TH, Yoon HK, Jung SJ, Park JS, et al. Value of 18F-FDG PET/CT for early prediction of pathologic response (by residual cancer burden criteria) of locally advanced breast cancer to neoadjuvant chemotherapy. Clin Nucl Med 2014;39:882-6.

19. Li Y, Qian ZJ, Ryu B, Lee SH, Kim MM, Kim SK. Chemical components and its antioxidant properties in vitro: an edible marine brown alga, Ecklonia cava. Bioorg Med Chem 2009;17:1963-73.

20. Heo SJ, Ko SC, Cha SH, Kang DH, Park HS, Choi YU, et al. Effect of phlorotannins isolated from Ecklonia cava on melanogenesis and their protective effect against photo-oxidative stress induced by 
UV-B radiation. Toxicol In Vitro 2009;23:1123-30.

21. Nagayama K, Iwamura Y, Shibata T, Hirayama I, Nakamura T. Bactericidal activity of phlorotannins from the brown alga Ecklonia kurome. J Antimicrob Chemother 2002;50:889-93.

22. Shin HC, Hwang HJ, Kang KJ, Lee BH. An antioxidative and antiinflammatory agent for potential treatment of osteoarthritis from Ecklonia cava. Arch Pharm Res 2006;29:165-71.

23. Joe MJ, Kim SN, Choi HY, Shin WS, Park GM, Kang DW, et al. The inhibitory effects of eckol and dieckol from Ecklonia stolonifera on the expression of matrix metalloproteinase- 1 in human dermal fibroblasts. Biol Pharm Bull 2006;29:1735-9.

24. Elmore S. Apoptosis: a review of programmed cell death. Toxicol Pathol 2007;35:495-516
25. Hengartner MO. The biochemistry of apoptosis. Nature 2000;407: $770-6$.

26. MacLachlan TK, Sang N, Giordano A. Cyclins, cyclin-dependent kinases and cdk inhibitors: implications in cell cycle control and cancer. Crit Rev Eukaryot Gene Expr 1995;5:127-56.

27. Hartwell LH, Weinert TA. Checkpoints: controls that ensure the order of cell cycle events. Science 1989;246:629-34.

28. Sherr CJ, Roberts JM. Inhibitors of mammalian G1 cyclin-dependent kinases. Genes Dev 1995;9:1149-63.

29. Luk SC, Siu SW, Lai CK, Wu YJ, Pang SF. Cell cycle arrest by a natural product via G2/M checkpoint. Int J Med Sci 2005;2:64-9.

30. Hartwell LH, Kastan MB. Cell cycle control and cancer. Science 1994;266:1821-8. 